Pacific Journal of Mathematics

ON THE ROLE OF AN ABELIAN PHASE GROUP IN
RELATIVIZED PROBLEMS IN TOPOLOGICAL DYNAMICS 


\title{
ON THE ROLE OF AN ABELIAN PHASE GROUP IN RELATIVIZED PROBLEMS IN TOPOLOGICAL DYNAMICS
}

\author{
Douglas McMaHON
}

\begin{abstract}
In this paper we are concerned with transformation groups having compact Hausdorff phase space. This paper is an outgrowth of an example that answers negatively the well-known question, must a proximally equicontinuous minimal set with abelian phase group be locally almost periodic; see Examples 3.1 and 3.3 .
\end{abstract}

In $\S 2$ we associate with each countable or finite group $T$ a function $F$ from $X \times S$ onto $T$ such that $F\left(x, s s^{\prime}\right)=F(x, s) F\left(x s, s^{\prime}\right)$, where $X=$ $\amalg_{1}^{\infty}\{0,1\}$ and $S$ is the direct sum $\amalg_{1}^{\infty}\{0,1\}$ given the discrete topology. And we associate with each transformation group $(Y, T)$ the extension $(X \times Y, S)$ of $(X, S)$ defined by $(x, y) s=(x s, y F(x, s))$. If $(Y, T)$ is minimal, then $(X \times Y, S)$ is minimal; and if in addition, $\varphi:(Y, T) \rightarrow(W, T)$ is a homomorphism, then $\psi=$ identity $\times \varphi:(X \times Y, S) \rightarrow(X \times W, S)$ is a homomorphism and shares many of the dynamical and topological properties of $\varphi$. For example, $\psi$ is proximal iff $\varphi$ is and $\psi$ is distal iff $\varphi$ is. This indicates that for many relativized problems one may assume that the phase group is abelian (or indeed $\amalg\{0,1\}$ ) without loss of generality. There are a great many properties that $\varphi$ and $\psi$ may share, we study some of those properties that are of particular importance in the structure of minimal sets and homomorphisms in general.

The association we use in $\$ 2$ is developed in a more general setting in $\S 1$. This general setting allows us to give an example of a proximally equicontinuous minimal set with abelian phase group and connected phase space that is not locally almost periodic, see Example 3.3.

I would like to thank T. S. Wu for his helpful suggestions.

This paper was announced in [4], where a slightly different point of view is taken.

Definitions and Notation. Suppose $\varphi:(X, T) \rightarrow(Y, T)$ is a homomorphism of minimal sets. Define $R(\varphi)=\left\{\left(x, x^{\prime}\right): \varphi(x)=\varphi\left(x^{\prime}\right)\right\}$. The relativized regionally proximal relation is $Q(\varphi)=\left\{\left(x, x^{\prime}\right)\right.$ : for some nets $\left(x_{n}, x_{n}^{\prime}\right) \in R(\varphi)$ and $t_{n}$ in $T, \lim \left(x_{n}, x_{n}^{\prime}\right) t_{n}=(x, x)$ and $\lim \left(x_{n}, x_{n}^{\prime}\right)=$ $\left.\left(x, x^{\prime}\right)\right\}$; the relativized proximal relation is $P(\varphi)=R(\varphi) \cap P_{X}$, where $P_{X}=P=\left\{\left(x, x^{\prime}\right)\right.$ : for some net $\left.t_{n}, \lim x t_{n}=\lim x^{\prime} t_{n}\right\}$ is the proximal 
relation on $X$; the regionally proximal relation on $X, Q_{X}=Q$ equals $Q(\varphi)$ when $Y$ is a singleton. The relativized distal structure relation, $S_{d}(\varphi)$, and the relativized equicontinuous structure relation, $S_{e}(\varphi)=$ $S(\varphi)$, are the smallest closed invariant equivalence relations containing $P(\varphi)$ and $Q(\varphi)$ respectively. We say $\varphi$ is proximal iff $R(\varphi) \subset P_{X} . \varphi$ is distal iff $R(\varphi) \cap P_{X}=P(\varphi)=\Delta$, the diagonal of $X$. A closed invariant equivalence relation, $R$, on $X$ is called proximal or distal if the induced homomorphism $(X, T) \rightarrow(X / R, T)$ is; so $R$ is a proximal equivalence relation iff $R \subset P_{X}$, and $R$ is a distal equivalence relation iff $R \cap P_{X}=\Delta$.

1. The basic idea for the construction in this section is as follows. Given homeomorphisms $\alpha, \beta$ of a compact Hausdorff space $Y$, take two copies of $Y$, (i.e. $\{0,1\} \times Y$ ), and define homeomorphism $\theta_{\alpha}$ and $\theta_{\beta}$ on $\{0,1\} \times Y$ by $(0, y) \theta_{\alpha}=(1, y \alpha),(1, y) \theta_{\alpha}=\left(0, y \alpha^{-1}\right),(0, y) \theta_{\beta}=(0, y \beta)$, and $(1, y) \theta_{\beta}=\left(1, y \alpha^{-1} \beta \alpha\right)$. One can check that $\theta_{\beta} \theta_{\alpha}=\theta_{\alpha} \theta_{\beta}$.

One could take the more general approach of assuming $\theta_{\alpha}$ was defined by $(0, y) \theta_{\alpha}=(1, y \alpha),(1, y) \theta_{\alpha}=\left(0, y \alpha^{\prime}\right)$ for some homeomorphism $\alpha^{\prime}$ of $Y$. Now with $\theta_{\beta}$ defined so that $\theta_{\beta}(0, y)=(0, y \beta)$, in order that $\theta_{\alpha} \theta_{\beta}=\theta_{\beta} \theta_{\alpha}$ one must define,

$$
\begin{aligned}
(1, y) \theta_{\beta} & =(1, y) \theta_{\alpha}^{-1} \theta_{\beta} \theta_{\alpha}=\left(0, y \alpha^{-1}\right) \theta_{\beta} \theta_{\alpha} \\
& =\left(0, y \alpha^{-1} \beta\right) \theta_{\alpha}=\left(1, y \alpha^{-1} \beta \alpha\right)
\end{aligned}
$$

Then $(1, y) \theta_{\beta} \theta_{\alpha}^{-1}=(1, y) \theta_{\alpha}^{-1} \theta_{\beta}$. .Also

$$
(0, y) \theta_{\beta} \theta_{\alpha}^{-1}=(0, y \beta) \theta_{\alpha}^{-1}=\left(1, y \beta \alpha^{\prime-1}\right)
$$

while $(0, y) \theta_{\alpha}^{-1} \theta_{\beta}=\left(1, y \alpha^{\prime-1}\right) \theta_{\beta}=\left(1, y \alpha^{\prime-1} \alpha^{-1} \beta \alpha\right)$. So $\theta_{\alpha} \theta_{\beta}=\theta_{\beta} \theta_{\alpha} \quad$ iff $\beta \alpha^{\prime-1}=\alpha^{\prime-1} \alpha^{-1} \beta \alpha$ iff $\beta \alpha \alpha^{\prime}=\alpha \alpha^{\prime} \beta$. So $\alpha \alpha^{\prime}=\gamma$ in a homeomorphism of $Y$ that commutes with $\beta$, that is, $\alpha^{\prime}=\alpha^{-1} \gamma$ differs from $\alpha^{-1}$ by a homeomorphism that commutes with $\beta$. Conversely if $\gamma$ is a homeomorphism of $Y$ that commutes with $\beta$, define $(0, y) \theta_{\gamma}=(0, y \gamma)$, $(1, y) \theta_{\gamma}=(1, y)$, define $(0, y) \theta_{\alpha}^{*}=(1, y \alpha),(1, y) \theta_{\alpha}^{*}=\left(1, y \alpha^{-1}\right)$, and define $(0, y) \theta_{\beta}=(0, y \beta),(1, y) \theta_{\beta}=\left(1, y \alpha^{-1} \beta \alpha\right)$, then $\theta_{\beta} \theta_{\alpha}^{*}=\theta_{\alpha}^{*} \theta_{\beta}, \theta_{\alpha}=\theta_{\alpha}^{*} \theta_{\gamma}$ and $\theta_{\beta} \theta_{\alpha}=\theta_{\beta} \theta_{\alpha}^{*} \theta_{\gamma}=\theta_{\alpha}^{*} \theta_{\beta} \theta_{\gamma}=\theta_{\alpha}^{*} \theta_{\gamma} \theta_{\beta}=\theta_{\alpha} \theta_{\beta}$. Thus the general, $\theta_{\alpha}$, is easily derived from the more special, $\theta_{\alpha}^{*}$; we will not consider this particular generalization in what follows.

To generalize from two homeomorphisms to a countable number, consider a countable set $\left\{\alpha_{n}\right\}_{1}^{\infty}$ of homeomorphisms of $Y$ and let $X=\Pi_{1}^{\infty}\{0,1\}$. Define $\theta_{1}: X \times Y \rightarrow X \times Y \quad$ by $\left(\left(0, l_{2}, \cdots\right), y\right) \theta_{1}=$ $\left(\left(1, l_{2}, l_{3}, \cdots\right), y \alpha_{1}\right)$ and $\left(\left(1, l_{2}, \cdots\right), y\right) \theta_{1}=\left(\left(0, l_{2}, \cdots\right), y \alpha_{1}^{-1}\right)$. Then define $\theta_{2}$ by 


$$
\begin{aligned}
& \left(\left(0,0, l_{3}, \cdots\right), y\right) \theta_{2}=\left(\left(0,1, l_{3}, \cdots\right), y \alpha_{2}\right) \\
& \left(\left(0,1, l_{3}, \cdots\right), y\right) \theta_{2}=\left(\left(0,0, l_{3}, \cdots\right), y \alpha_{2}^{-1}\right) \\
& \left(\left(1,0, l_{3}, \cdots\right), y\right) \theta_{2}=\left(\left(1,1, l_{3}, \cdots\right), y \alpha_{1}^{-1} \alpha_{2} \alpha_{1}\right) \\
& \left(\left(1,1, l_{3}, \cdots\right), y\right) \theta_{2}=\left(\left(1,0, l_{3}, \cdots\right), y \alpha_{1}^{-1} \alpha_{2}^{-1} \alpha_{1}\right) .
\end{aligned}
$$

One would define $\theta_{3}$ similarly, for example

$$
\begin{aligned}
& \left(\left(0,0,0, l_{4}, \cdots\right), y\right) \theta_{3}=\left(\left(0,0,1, l_{4}, \cdots\right), y \alpha_{3}\right) \\
& \left(\left(0,0,1, l_{4}, \cdots\right), y\right) \theta_{3}=\left(\left(0,0,0, l_{4}, \cdots\right), y \alpha_{3}^{-1}\right) \\
& \left(\left(1,0,0, l_{4}, \cdots\right), y\right) \theta_{3}=\left(\left(1,0,1, l_{4}, \cdots\right), y \alpha_{1}^{-1} \alpha_{3} \alpha_{1}\right) \\
& \left(\left(1,1,0, l_{4}, \cdots\right), y\right) \theta_{3}=\left(\left(1,1,1, l_{4}, \cdots\right), y \alpha_{1}^{-1} \alpha_{2}^{-1} \alpha_{3} \alpha_{2} \alpha_{1}\right) .
\end{aligned}
$$

For each $n, \theta_{n}$ could be defined similarly and one could check that $\theta_{n} \theta_{m}=\theta_{m} \theta_{n}$.

To generalize it one step further, for $n=1,2, \cdots$, let $G_{n}$ be a compact Hausdorff topological group and for each $n$ let $\left(G_{n} \times Y, G_{n}\right)$ be a transformation group such that $(g, y) h=\left(g h, \psi_{n}(g, y, h)\right)$ where $g, h \in G_{n}, y \in Y$, and $\psi_{n}$ is the projection onto $Y$ of $(g, y) h$. (To insure the continuity of the action of each $s$ in $S$ on $X$ defined below, the phase group $G_{n}$ must retain its topology in the above.) Let $e$ denote the identity of $G_{n}$ for all $n$, let $X=\prod_{n=1}^{\infty} G_{n}$, and let $x_{n}^{h} \in X$ be defined by $x_{n}^{h}(n)=h$ and $x_{n}^{h}(i)=e$ if $i \neq n$. Extend the action of $G_{n}$ on $G_{n} \times Y$ to $X \times Y$ by $(x, y) h=\left(x x_{n}^{h}, \psi_{n}\left(g_{n}, y, h\right)\right)$ where $g_{n}$ is the $n$th coordinate of $x$ and $h \in G_{n}$.

Now define $(x, y) \theta_{n}^{h}=(x, y) g_{1}^{-1} g_{2}^{-1} \cdots g_{n-1}^{-1} h g_{n-1} \cdots g_{1}$ where $x=$ $\left(g_{1}, g_{2}, \cdots\right)$ and $h \in G_{n}$. Note the projection of $(x, y) \theta_{n}^{h}$ onto $X$ is $x x_{n}^{h}$.

If we let $G_{n}=Z_{2}$ and the action of $\left(G_{n} \times Y, G_{n}\right)$ is defined by $(0, y) 0=(0, y),(1, y)=(1, y),(0, y) 1=(1, y \alpha)$, and $(1, y) 1=\left(0, y \alpha^{-1}\right)$, then we have the more specific construction mentioned above for a countable number of homeomorphisms with $\theta_{n}=\theta_{n}^{1}$.

We now show that $\theta_{n}^{h} \theta_{m}^{f}=\theta_{m}^{f} \theta_{n}^{h}$ where $f \in G_{m}, h \in G_{n}$, and $m \neq n$. Let $m>n$, then

$$
\begin{aligned}
\left((x, y) \theta_{n}^{h}\right) \theta_{m}^{f}= & \left((x, y) \theta_{n}^{h}\right) g_{1}^{-1} \cdots g_{n-1}^{-1}\left(g_{n} h\right)^{-1} \cdots g_{m-1}^{-1} f g_{m-1} \cdots\left(g_{n} h\right) g_{n-1} \cdots g_{1} \\
= & (x, y) g_{1}^{-1} \cdots g_{n-1}^{-1} h g_{n-1} \cdots g_{1} g_{1}^{-1} \cdots g_{n-1}^{-1}\left(g_{n} h\right)^{-1} \\
& \cdots g_{m-1}^{-1} f g_{m-1} \cdots\left(g_{n} h\right) g_{n-1} \cdots g_{1} \\
= & (x, y) g_{1}^{-1} \cdots g_{n-1}^{-1} g_{n}^{-1} \cdots g_{m-1}^{-1} f g_{m-1} \cdots\left(g_{n} h\right) g_{n-1} \cdots g_{1} \\
= & (x, y) g_{1}^{-1} \cdots g_{m-1}^{-1} f g_{m-1} \cdots g_{n} g_{n-1} \cdots g_{1} g_{1}^{-1} \cdots g_{n-1}^{-1} h g_{n-1} \cdots g_{1} \\
= & (x, y) \theta_{m}^{f} \theta_{n}^{h} .
\end{aligned}
$$


Now clearly $\theta_{n}^{h}$ is a homeomorphism of $X \times Y, \theta_{n}^{h} \theta_{n}^{f}=\theta_{n}^{h f}, \theta_{n}^{e}$ is the identity, and $\left(\theta_{n}^{h}\right)^{-1}=\theta_{n}^{h^{-1}}$. The group generated by the $\left\{\theta_{n}^{h}\right\}$ is group isomorphic to the direct sum $\amalg G_{n}=\left\{x \in X: x=\left(g_{1}, g_{2}, \cdots\right), g_{n}=e\right.$ except for finitely many $n$ 's $\}$. Let $S=\amalg G_{n}$ be given the discrete topology, then $(X \times Y, S)$ is a transformation group, where $s \in S$ acts on $(x, y)$ by $(x, y) s=(x, y) \theta_{n_{1}}^{g_{1}} \cdots \theta_{n_{k}}^{g_{k}}$ where $s=x_{n_{1}}^{g_{1}} \cdots x_{n_{k}}^{g_{k}}$.

Note the projection $\Pi$ of $X \times Y$ onto $X$ is an open homomorphism of $(X \times Y, S)$ onto $(X, S) ;(X, S)$ is an equicontinuous minimal set; and if $G_{n}$ is abelian for all $n$, then $S$ is abelian. Also for fixed $x$ and $s$, $F(x, s): Y \rightarrow Y$ defined by the equality $(x s, y F(x, s))=(x, y) s$ is a homeomorphism of $Y$ and for $s, s^{\prime} \in S, F(x, s) F\left(x s, s^{\prime}\right)=F\left(x, s s^{\prime}\right)$ as it must for $(X \times Y, S)$ to be a transformation group.

2. From 2.2 onward, we are considering minimal transformation groups only.

Let $(Y, T)$ be a transformation group with $T$ countable or finite. Let $\left\{t_{n}\right\}_{n \in N}$ be an enumeration of the elements of $T$ with each $t$ in $T$ repeated an infinite number of times, i.e. $\left\{n: t_{n}=t\right\}$ is infinite for each $t$ in $T$. Now let $G_{n}=Z_{2}=\{0,1\}, 1+1=0$, and let the action of $G_{n}$ on $G_{n} \times Y$ be $(0, y) 0=(0, y), \quad(1, y) 0=(1, y), \quad(0, y) 1=\left(1, y t_{n}\right), \quad(1, y) 1=$ $\left(0, y t_{n}^{-1}\right)$. Define $(X \times Y, S)$ as in $\S 1$ and note that the action is $(x, y) s=$ $(x s, y F(x, s))$ and $F(x, s) \in T$. This is clear since if $x=(x(1), x(2), \cdots)$ and $x_{n}^{1}$ is the element of $S$ defined by $x_{n}^{1}(i)=0$ if $i \neq n, x_{n}^{1}(n)=1$, then $(x, y) x_{n}^{1}=\left(x x_{n}^{1}, y t_{1}^{-x(1)} \cdots t_{n-1}^{-x(n-1)} t_{n}^{*} t_{n-1}^{x(n-1)} \cdots t_{1}^{x(1)}\right)$ where $t_{n}^{*}=t_{n}$ if $x(n)=0$, $t_{n}^{*}=t_{n}^{-1}$ if $x(n)=1$. (Clearly $F$ is independent of $Y$.)

Now let $(W, T)$ be a transformation group and form $(X \times W, S)$ in the same manner as $(X \times Y, S)$ was formed (i.e. take $\left\{t_{n}\right\}$ to be the same enumeration - take the same $F)$. If $\varphi:(Y, T) \rightarrow(W, T)$ is a homomorphism, then it is easy to show that $\psi:(X \times Y, S) \rightarrow(X \times W, S)$ defined by $\psi(x, y)=(x, \varphi(Y))$ is a homomorphism. Note for $x \in X$, $\left(y, y^{\prime}\right) \in R(\varphi)$ iff $\left((x, y),\left(x, y^{\prime}\right)\right) \in R(\psi)$. We will show that there is a very close relationship between $\varphi$ and $\psi$.

Suppose $x \in S$ and $x(n)=0$ for $n>M$, then for $n>M$ the action of $x_{n}^{1} \in S$ on $(x, y)$ is $(x, y) x_{n}^{1}=\left(x x_{n}^{1}, y \hat{t}^{-1} t_{n} \hat{t}\right)$ where $\hat{t}=t_{M}^{x(M)} \cdots t_{2}^{x(2)} t_{1}^{x(1)}$. Suppose in addition that $t \in T$, and consider $\hat{t} t \hat{t}^{-1}$, since $\hat{t} t \hat{t}^{-1}=t_{n}$ for some increasing sequence of $n$ 's in $N$ with $n>M$, it is clear that for $y \in Y$, $(x, y t) \in \operatorname{cls}[(x, y) S]$. Thus we have the following lemma.

Lemma 2.1. Let $x \in S, t \in T$, then for some net $s_{\lambda}$ in $S$, and for all $y$ in $Y,(x, y t)=\lim (x, y) s_{\lambda}$.

Note from this it is clear that $(X \times Y, S)$ is minimal, if $(Y, T)$ is. For the remainder of this section we are considering only minimal sets. 
Proposition 2.2.

(I) For $x, x^{\prime} \in X$, if $\left((x, y),\left(x^{\prime} y^{\prime}\right)\right) \in P_{(X \times Y, S)}$, then $x=x^{\prime}$ and $\left(y, y^{\prime}\right) \in P_{(Y, T)}$.

(ii) For $x \in S$, if $\left(y, y^{\prime}\right) \in P_{(Y, T)}$, then $\left((x, y),\left(x, y^{\prime}\right)\right) \in P_{(X \times Y, S)}$.

Proof. If $\quad\left((x, y),\left(x^{\prime}, y^{\prime}\right)\right) \in P_{(X \times Y, S)}, \quad$ then $\quad\left(x, x^{\prime}\right)=$ $\Pi \times \Pi\left((x, y),\left(x^{\prime}, y^{\prime}\right)\right) \in P_{(X, S)}=\Delta$ and thus $x=x^{\prime}$. And since action by $s$ in $S$ has the effect on the $Y$-coordinate of an action by some $t$ in $T$, we have that $\left(y, y^{\prime}\right) \in P_{(Y, T)}$.

The second statement for $x \in S$ follows from Lemma 2.1.

COROllary 2.3. $\varphi$ is a distal homomorphism iff $\psi$ is.

Proposition 2.4. $\varphi$ is a proximal homomorphism iff $\psi$ is.

Proof. Suppose $R(\varphi)=P(\varphi)$. If $\left((x, y),\left(x^{\prime}, y^{\prime}\right)\right) \in R(\psi)$, then $x=$ $x^{\prime}$ and so we may take a net $s_{\lambda}$ with $\left((x, y),\left(x^{\prime}, y^{\prime}\right)\right) s_{\lambda} \rightarrow\left((e, \hat{y}),\left(e, \hat{y}^{\prime}\right)\right) \in$ $R(\psi)$ for some $\hat{y}, \hat{y}^{\prime} \in Y$. Now $\left(\hat{y}, \hat{y}^{\prime}\right) \in R(\varphi)=P(\varphi)$ and $e \in S$, so $\left((e, \hat{y}),\left(e \hat{y}^{\prime}\right)\right) \in P(\psi)$. This implies $\left((x, y),\left(x^{\prime}, y^{\prime}\right)\right) \in P(\psi),\left(x=x^{\prime}\right)$, and thus $R(\psi)=P(\psi)$ and $\psi$ is a proximal homomorphism.

Now suppose $R(\psi)=P(\psi)$ and $\left(y, y^{\prime}\right) \in R(\varphi)$. Then $\left((e, y),\left(e, y^{\prime}\right)\right) \in$ $R(\psi)=P(\psi)$. Then by $2.2,\left(y, y^{\prime}\right) \in P(\varphi)$.

Proposition 2.5. For each $x \in X, \quad\left(y, y^{\prime}\right) \in Q(\varphi) \quad$ iff $\left((x, y),\left(x, y^{\prime}\right)\right) \in Q(\psi)$.

Proof. If $\left(y, y^{\prime}\right) \in Q(\varphi)$, then for any neighborhoods $U$ of $x, V$ of $y$, and $V^{\prime}$ of $y^{\prime}$ there exists $x^{\prime} \in S \cap U,\left(\hat{y}, \hat{y}^{\prime}\right) \in V \times V^{\prime} \cap R(\varphi)$, and $t \in T$ such that $\hat{y} t \in V$ and $\hat{y}^{\prime} t \in V$. Now by 2.1 there exists $s \in S$ with $\left(\left(x^{\prime}, \hat{y}\right),\left(x^{\prime}, \hat{y}^{\prime}\right)\right) s \in(U \times V) \times(U \times V) \quad$ while $\quad\left(\left(x^{\prime}, \hat{y}\right),\left(x^{\prime}, \hat{y}^{\prime}\right)\right) \in$ $(U \times V) \times\left(U \times V^{\prime}\right) \cap R(\psi)$, thus $\left((x, y),\left(x, y^{\prime}\right)\right) \in Q(\psi)$.

If $\left((x, y),\left(x, y^{\prime}\right)\right) \in Q(\psi)$, then since an action by an $s$ in $S$ has the effect on the $Y$-coordinate of an action by some $t$ in $T$, it is clear that $\left(y, y^{\prime}\right) \in Q(\varphi)$.

PROPOSITION 2.6.

(i) For $x$ in $X,\left(y, y^{\prime}\right) \in S_{d}(\varphi)$ iff $\left((x, y),\left(x, y^{\prime}\right)\right) \in S_{d}(\psi)$.

(ii) For $x$ in $X,\left(y, y^{\prime}\right) \in S_{e}(\varphi)$ iff $\left((x, y),\left(x, y^{\prime}\right)\right) \in S_{e}(\psi)$.

Proof.

(i) $S_{d}(\varphi)$ is generated by letting $C_{1}(\varphi)=\operatorname{cls} P(\varphi), \quad E_{1}(\varphi)=$ $E\left(C_{1}(\varphi)\right)$, where $E(C)$ is the smallest equivalence relation containing $C, C_{2}(\varphi)=\operatorname{cls} E_{1}, E_{2}(\varphi)=E\left(C_{2}(\varphi)\right)$, and in general for any ordinal $\lambda, C_{\lambda}(\varphi)=\operatorname{cls}\left(\cup\left\{E_{\mu}(\varphi): \mu<\lambda\right\}\right), E_{\lambda}(\varphi)=E\left(C_{\lambda}(\varphi)\right)$. This gives a chain of sets which ends when $C_{\lambda}(\varphi)=E_{\lambda}(\varphi)=S_{d}(\varphi)$.

First note that if $\left((x, y),\left(x^{\prime}, y^{\prime}\right)\right) \in S_{d}(\psi)$, then $x=x^{\prime}$. By 2.2, for all $x$ 
in $X,\left(y, y^{\prime}\right) \in \operatorname{cls} P(\varphi)$ iff $\left((x, y),\left(x, y^{\prime}\right)\right) \in \operatorname{cls} P(\psi)$; that is for all $x$ in $X$, $\left(y, y^{\prime}\right) \in C_{1}(\phi)$ iff $\left((x, y),\left(x, y^{\prime}\right)\right) \in C_{1}(\psi)$. Suppose that for $\mu<\lambda$ we have, for all $x$ in $X,\left(y, y^{\prime}\right) \in C_{\mu}(\phi)$ iff $\left((x, y),\left(x, y^{\prime}\right)\right) \in C_{\mu}(\psi)$. It follows easily that for $\mu<\lambda$ and for all $x$ in $X,\left(y, y^{\prime}\right) \in E_{\mu}(\phi)$ iff $\left((x, y),\left(x, y^{\prime}\right)\right) \in E_{\mu}(\psi)$ and then for all $x$ in $X,\left(y, y^{\prime}\right) \in C_{\lambda}(\phi)$ iff $\left((x, y),\left(x, y^{\prime}\right)\right) \in C_{\lambda}(\psi)$. Thus (i) follows.

(ii) Is proved in a similar manner.

Corollary 2.7. $S(\varphi)=R(\varphi)$ iff $S(\psi)=R(\psi)$.

Proposition 2.8. For $x \in X,\left(y, y^{\prime}\right)$ is an almost periodic point in $(Y \times Y, T)$ iff $\left((x, y),\left(x, y^{\prime}\right)\right)$ is an almost periodic point in $((X \times Y) \times$ $(X \times Y), S)$.

Proof. Suppose $\left(y, y^{\prime}\right)$ is an almost periodic point in $R(\varphi)$. First we show that $\left((e, y),\left(e, y^{\prime}\right)\right)$ is an almost periodic point. If $\left((x, \hat{y}),\left(x, \hat{y}^{\prime}\right)\right) \in$ $\operatorname{cls}\left(\left((e, y),\left(e, y^{\prime}\right)\right) S\right)$, let $s_{\lambda}$ be a net in $S$ with $\left((e, \bar{y}),\left(e, \bar{y}^{\prime}\right)\right)=$ $\lim \left((x, \hat{y}),\left(x, \hat{y}^{\prime}\right)\right) s_{\lambda}$ for some $\bar{y}, \bar{y}^{\prime}$ in $Y$, then $\left(\bar{y}, \bar{y}^{\prime}\right) \in \operatorname{cls}\left(\left(y, y^{\prime}\right) T\right)$, since $S$ acts on the $Y$-coordinate by elements of $T$. So $\left(y, y^{\prime}\right) \in \operatorname{cls}\left(\left(\bar{y}, \bar{y}^{\prime}\right) T\right)$. Let $U, V, V^{\prime}$ be neighborhoods of $e, y, y^{\prime}$ respectively. Then by 2.1 for some $s \in S, \quad\left((e, \bar{y}),\left(e, \bar{y}^{\prime}\right)\right) s \in(U \times V) \times\left(U \times V^{\prime}\right) \quad$ and so for some $\lambda$, $\left((x, \hat{y}),\left(x, \hat{y}^{\prime}\right)\right) s_{\lambda} s \in(U \times V) \times\left(U \times V^{\prime}\right) . \quad$ So $\quad\left((e, y),\left(e, y^{\prime}\right)\right) \in$ $\operatorname{cls}\left(\left((x, \hat{y}),\left(x, \hat{y}^{\prime}\right)\right) S\right)$ and is therefore an almost periodic point.

Now for $d \in S \subseteq X$ and any $y^{*} \in Y,\left(e, y^{*}\right) d=\left(d, y^{*} t\right)$ where $t \in T$ depends on $d$. By 2.1, $\left(\left(e, y t^{-1}\right),\left(e, y^{\prime} t^{-1}\right)\right) \in \operatorname{cls}\left(\left((e, y),\left(e, y^{\prime}\right)\right) S\right)$ and so $\left((d, y),\left(d, y^{\prime}\right)\right)=\left(\left(e, y^{\prime} t^{-1}\right),\left(e, y^{\prime} t^{-1}\right)\right) d \in \operatorname{cls}\left(\left((e, y),\left(e, y^{\prime}\right)\right) S\right)$. And since $S$ is dense in $X,\left((x, y),\left(x, y^{\prime}\right)\right) \in \operatorname{cls}\left(\left((e, y),\left(e, y^{\prime}\right)\right) S\right)$ for $x \in X$ and so is an almost periodic point.

Now suppose $\left((x, y),\left(x, y^{\prime}\right)\right)$ is an almost periodic point in $R(\psi)$. We will first show $\left((e, y),\left(e, y^{\prime}\right)\right) \in \operatorname{cls}\left(\left((x, y),\left(x, y^{\prime}\right)\right) S\right)$ and so is an almost periodic point in $R(\psi)$.

For some $\hat{y}, \hat{y}^{\prime}$ in $Y,\left((e, \hat{y}),\left(e, \hat{y}^{\prime}\right)\right) \in \operatorname{cls}\left(\left((x, y),\left(x, y^{\prime}\right)\right) S\right)$. Then $\left((x, y),\left(x, y^{\prime}\right)\right) \in \operatorname{cls}\left(\left((e, \hat{y}),\left(e, \hat{y}^{\prime}\right)\right) S\right)$. Now let $U, V, V^{\prime}$ be neighborhoods of $e, y, y^{\prime}$ respectively. Then for some $s \in S,\left((e, \hat{y}),\left(e, \hat{y}^{\prime}\right)\right) s \in(X \times V) \times$ $\left(X \times V^{\prime}\right)$ and $\left((e, \hat{y}),\left(e, \hat{y}^{\prime}\right)\right) s=\left(\left(s, \hat{y} t^{*}\right),\left(s, \hat{y}^{\prime} t^{*}\right)\right)$ for some $t^{*}$ in $T$. For some integer $M, x_{n}^{1} \in U$ for $n>M$, so take $n>M$ with $t_{n}=t^{*}$, then $\quad\left((e, \hat{y}),\left(e, \hat{y}^{\prime}\right)\right) x_{n}^{1} \in(U \times V) \times\left(U \times V^{\prime}\right)$. So $\quad\left((e, y),\left(e, y^{\prime}\right)\right) \in$ $\operatorname{cls}\left(\left((x, y),\left(x, y^{\prime}\right)\right) S\right)$ and so is an almost periodic point.

Now suppose $\left((e, y),\left(e, y^{\prime}\right)\right)$ is an almost periodic point and $\left(\hat{y}, \hat{y}^{\prime}\right) \in$ $\operatorname{cls}\left(\left(y, y^{\prime}\right) T\right)$. Then by 2.1, $\left((e, \hat{y}),\left(e, \hat{y}^{\prime}\right)\right) \in \operatorname{cls}\left(\left((e, y),\left(e, y^{\prime}\right)\right) S\right)$. So $\left((e, y),\left(e, y^{\prime}\right)\right) \in \operatorname{cls}\left(\left((e, \hat{y}),\left(e, \hat{y}^{\prime}\right)\right) S\right)$ and therefore $\left(y, y^{\prime}\right) \in \operatorname{cls}\left(\left(\hat{y}, \hat{y}^{\prime}\right) T\right)$. Thus $\left(y, y^{\prime}\right)$ is an almost periodic point.

COROllary 2.9. The almost periodic points in $(R(\varphi), T)$ are dense in $R(\varphi)$ iff the almost periodic points in $(R(\psi), S)$ are dense in $R(\psi)$. 
We note that the restriction in 2.2 (ii) that $x$ be an element of $S$ is necessary. For example let $(Y, T)$ be a minimal set with $T=$ integers and for some $\left(y, y^{\prime}\right) \in P_{(Y, T)}$ and for some index $\alpha$ on $Y$, the set of negative integers is contained in the set $\left\{t:\left(y, y^{\prime}\right) t \notin \alpha\right\}$ (see [6] for an example of such a space). Now define $x$ by

$$
x(n)=\left\{\begin{array}{lll}
0 & \text { if } & t_{n} \leqq 0 \\
1 & \text { if } & t_{n}>0
\end{array}\right.
$$

Then $\left((x, y),\left(x, y^{\prime}\right)\right) \notin P_{(X \times Y, S)}$ since the effect on the $Y$-coordinate is only that of negative integers. Note that in view of 2.4 , the smallest invariant closed equivalence relation on $(Y, T)$ containing $\left(y, y^{\prime}\right)$ is not proximal (i.e. is not contained in $P_{(Y, T)}$ ).

Up to now we have looked at a homomorphism $\varphi:(Y, T) \rightarrow(W, T)$ and the corresponding homomorphism $\psi=i d \times \varphi$ : $(X \times Y, S) \rightarrow(X \times W, S)$. Now we will consider a minimal set $(Z, S)$ such that the diagram

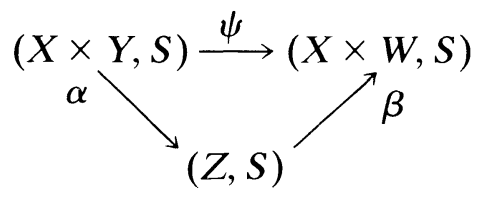

commutes. Now $R(\varphi)=\left\{\left(y, y^{\prime}\right): \varphi(y)=\varphi\left(y^{\prime}\right)\right\}, R(\psi)=\left\{\left((x, y),\left(x, y^{\prime}\right)\right)\right.$ : $\left.x \in X,\left(y, y^{\prime}\right) \in R(\varphi)\right\}$, and $R(\alpha) \subseteq R(\psi)$. This situation is treated in the following proposition, note $(W, T)$ is isomorphic to $(Y / R(\varphi), T)$ and $(X \times W, S)$ is isomorphic to $(X \times Y / R(\psi), S)$.

Proposition 2.10. Suppose $R$ is a closed invariant equivalence relation contained in $R(\psi)$. Then the set $R^{\prime}=\left\{\left(y, y^{\prime}\right):\left((e, y),\left(e, y^{\prime}\right)\right) \in R\right\}$ is a closed invariant equivalence relation contained in $R(\varphi)$ and the associated equivalence relation $R^{*}=\left\{\left((x, y),\left(x, y^{\prime}\right)\right):\left((e, y),\left(e, y^{\prime}\right)\right) \in R\right.$ (i.e. $\left.\left.\left(y, y^{\prime}\right) \in R^{\prime}\right)\right\}$ equals $\left\{\left((x, y),\left(x, y^{\prime}\right)\right):\left(\left(x^{\prime}, y\right),\left(x^{\prime}, y^{\prime}\right)\right) \in R\right.$ for every $x^{\prime}$ in $X\}$ and the natural homomorphism of $X \times Y / R^{*} \rightarrow X \times Y / R$ is such that the fiber over $(e, y) / R$ is a singleton, for $y$ in $Y$. Moreover for $s$ in $S, \quad R^{\prime}=\left\{\left(y, y^{\prime}\right):\left((s, y),\left(s, y^{\prime}\right)\right) \in R\right\} \quad$ and $\quad R^{*}=\left\{\left((x, y),\left(x, y^{\prime}\right)\right)\right.$ : $\left.\left((s, y),\left(s, y^{\prime}\right)\right) \in R\right\}$.

Proof. Fix $s$ in $S$, we first show that

$$
\begin{aligned}
\left\{\left((x, y),\left(x, y^{\prime}\right)\right):\right. & \left.\left((s, y),\left(s, y^{\prime}\right)\right) \in R\right\} \\
& =\left\{\left((x, y),\left(x, y^{\prime}\right)\right):\left(\left(x^{\prime}, y\right),\left(x^{\prime}, y^{\prime}\right)\right) \in R \text { for every } x^{\prime} \text { in } X\right\} .
\end{aligned}
$$


(Note both of these sets are closed.) Let $\left((s, y),\left(s, y^{\prime}\right)\right) \in R$. Then for $s^{\prime} \in S,\left((s, y),\left(s, y^{\prime}\right)\right) s^{\prime}=\left(\left(s s^{\prime}, y t\right),\left(s s^{\prime}, y^{\prime} t\right)\right) \in R S=R$ for some $t$ in $T$ and by 2.1

$$
\left(\left(s s^{\prime}, y\right),\left(s s^{\prime}, y^{\prime}\right)\right)=\left(\left(s s^{\prime}, y t t^{-1}\right),\left(s, s^{\prime}, y t t^{-1}\right)\right) \in \operatorname{cls} R=R .
$$

Since $S=s S$ is dense in $X,\left(\left(x^{\prime}, y\right),\left(x^{\prime}, y^{\prime}\right)\right) \in R$ for $x^{\prime}$ in $X$. So they are equal. Also for fixed $s$ in $S$, the set

$\left\{\left(y, y^{\prime}\right):\left((s, y),\left(s, y^{\prime}\right)\right) \in R\right\}=\left\{\left(y, y^{\prime}\right):\left((x, y),\left(x, y^{\prime}\right)\right)\right.$

$\in R$ for every $x$ in $X\}$.

To show that $R^{\prime}$ is invariant, let $t \in T$ and suppose $t_{n}=t^{-1}$. If $\left(y, y^{\prime}\right) \in R^{\prime}$, then $\quad\left((e, y),\left(e, y^{\prime}\right)\right) \in R$, so $\quad\left(\left(x_{n}^{1}, y\right),\left(x_{n}^{1}, y^{\prime}\right)\right) \in R \quad$ and $\left(\left(x_{n}^{1}, y\right),\left(x_{n}^{1}, y^{\prime}\right)\right) x_{n}^{1}=\left((e, y t),\left(e, y^{\prime} t\right)\right) \in R$; thus $\left(y t, y^{\prime} t\right) \in R^{\prime}$ and $R^{\prime}$ is invariant. Clearly then $R^{\prime}$ is a closed invariant equivalence relation.

Clearly $R^{*}$ is a closed invariant equivalence relation on $X \times Y$ and $R^{*} \subseteq R$. Now for $s$ in $S,\left((s, y),\left(s, y^{\prime}\right)\right) \in R^{*}$ iff $\left((s, y),\left(s, y^{\prime}\right)\right) \in R$, so the homomorphism $X \times Y / R^{*} \rightarrow X \times Y / R$ has the fiber over $(s, y) / R$ equal to $\left\{(s, y) / R^{*}\right\}$.

Corollary 2.11. If $R$ is a distal (closed invariant) equivalence relation, then $R=R^{*}$.

Now suppose $R_{1} \subseteq R_{2} \subseteq R(\psi)$ and consider the diagram

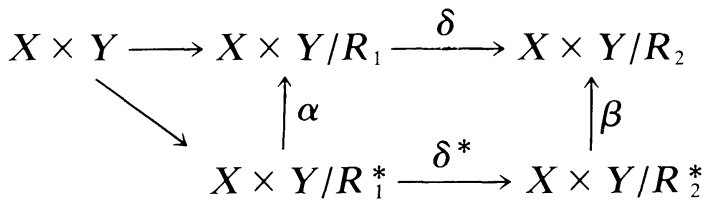

where $\alpha, \beta, \delta, \delta^{*}$ are the homomorphisms induced by the equivalence relations. Note that if $\psi$ is a distal homomorphism, then $R_{1}$ and $R_{2}$ are distal equivalence relations and so $R_{1}=R_{1}^{*}, R_{2}=R_{2}^{*}$ and $\delta=\delta^{*}$. Also clearly $\delta$ is a proximal homomorphism iff $\delta^{*}$ is. Considering $S$ as a subset of $X$ and any closed invariant equivalence relation $R \subseteq R(\psi)$, we see that the restriction of the induced map to $\left(S \times Y / R^{*}, S\right) \rightarrow(S \times Y / R, S)$ is an isomorphism and for $s$ in $S$ the map from $\{s\} \times Y / R \rightarrow Y / R^{\prime}$ defined by $(s, y) / R \rightarrow y / R^{\prime}$ is a homeomorphism of topological spaces.

Now suppose $\delta^{*}$ is open and let $\delta^{\prime}$ be the induced homomorphism from $\left(Y / R_{1}^{\prime}, T\right)$ onto $\left(Y / R_{2}^{\prime}, T\right)$, then $\delta^{\prime}$ is open and so $i d \times \delta^{\prime}: X \times$ $\left(Y / R_{1}^{\prime}\right) \rightarrow X \times\left(Y / R_{2}^{\prime}\right)$ is open, where $i d$ is the identity on $X$. We may identify $\delta^{*}$ with id $\times \delta^{\prime}$, since for $R \subseteq R(\psi)$ the map $\psi_{R}$ of $X \times\left(Y / R^{\prime}\right)$ onto $(X \times Y) / R$ defined by $\left(x, y / R^{\prime}\right) \mapsto(x, y) / R$ is an isomorphism. Then 
$\delta^{*}$ is open. This illustrates one approach to studying the topological properties of $\delta^{*}$ in comparison with $\delta$. Note that for the purpose of studying the relationship between $\delta$ and $\delta^{*}$ we may assume that $(X \times Y, S)$ is isomorphic to $\left(X \times Y / R_{1}^{*}, S\right)$, (since $\psi_{R}$ is an isomorphism). We now compare some of the dynamical properties of $\delta$ and $\delta^{*}$.

\section{Proposition 2.12. If $\delta$ is distal, then $\delta^{*}$ is distal.}

Proof. Note $\delta$ is distal iff $R_{2} \cap P \subseteq R_{1}$.

Now suppose $\left((x, y),\left(x^{\prime}, y\right)\right) \in R_{2}^{*} \cap P$. Then by definition of $R_{2}^{*}$ and by $2.2,\left((e, y),\left(e, y^{\prime}\right)\right) \in R_{2} \cap P \subseteq R_{1}$ and so $\left((x, y),\left(x, y^{\prime}\right)\right) \in R_{1}^{*}$. Then $R_{2}^{*} \cap P \subseteq R_{1}^{*}$ and $\delta^{*}$ is distal.

PROPOSITION 2.13. If the almost periodic points in $(R(\delta), S)$ are dense, then the almost periodic points in $\left(R\left(\delta^{*}\right), S\right)$ are dense.

Proof. Note $R(\delta \circ \alpha)=R_{2}$ and $R\left(\delta^{*}\right)=R_{2}^{*}$ because of our identification of $(X \times Y, S)$ with $\left(X \times Y / R_{1}^{*}, S\right)$. Let $\left((e, y),\left(e, y^{\prime}\right)\right) \in R(\delta \circ \alpha)$, so $\quad\left(\alpha(e, y), \alpha\left(e, y^{\prime}\right)\right) \in R(\delta)$. Now $(\alpha \times \alpha)^{-1}\left(\alpha \times \alpha\left((e, y),\left(e, y^{\prime}\right)\right)\right)=$ $\left\{\left((e, y),\left(e, y^{\prime}\right)\right)\right\}$ and since $\alpha \times \alpha$ is a closed map we have for any open neighborhood $U$ of $\left((e, y),\left(e, y^{\prime}\right)\right)$ there exists a neighborhood $V^{*}$ of $\alpha \times \alpha\left((e, y),\left(e, y^{\prime}\right)\right)$ with $V=(\alpha \times \alpha)^{-1} V^{*} \subseteq U$. Now there exists an almost periodic point in $V^{*} \cap R(\delta)$ and so there exists an almost periodic point, $\left((x, \bar{y}),\left(x, \bar{y}^{\prime}\right)\right)$, in $V \cap R(\delta \circ \alpha) \subset U \cap R(\delta \circ \alpha)=U \cap R_{2}$. As shown in the proof of $2.8,\left((e, \bar{y}),\left(e, \bar{y}^{\prime}\right)\right) \in \operatorname{cls}\left(\left((x, \bar{y}),\left(x, \bar{y}^{\prime}\right)\right) S\right)$ and so is an element of $R_{2}$. Thus $\left((x, y),\left(x, y^{\prime}\right)\right) \in R_{2}^{*}=R\left(\delta^{*}\right)$ and the almost periodic points are dense.

In working with the results of this section one should keep in mind that even if $(Y, T)$ and $(W, T)$ are not isomorphic, $(X \times Y, S)$ and $(X \times W, S)$ may be isomorphic. For example suppose $\alpha$ is a homeomorphism of $Y=W$ and for $T=$ integers, $(Y, T)$ is defined by $y n=\alpha^{n}(y)$ and $(W, T)$ by $w n=\alpha^{-n}(w)$. Then the map $(x, y) \mapsto\left(x x^{*}, y\right)$ is an isomorphism of $(X \times Y, S)$ onto $(X \times W, S)$ where $x^{*} \in X$ defined by $x^{*}(n)=1$ for all $n$. See [3] for an example in which $(Y, T)$ and $(W, T)$ are not isomorphic.

Now suppose $(X \times Y, S),(X \times W, S)$ are minimal sets, $(X$ and $S$ as usual) the first projections $\pi$ of $(X \times Y, S)$ onto $(X, S)$ and of $(X \times W, S)$ onto $(X, S)$ are homomorphisms, and $\alpha:(X \times Y, S) \rightarrow(X \times W, S)$ is a homomorphism. Then there is an isomorphism $\alpha^{*}:(X, S) \rightarrow(X, S)$ such that the diagram

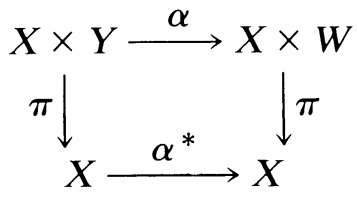


commutes, and $\alpha^{*}(x)$ equals the product of $\alpha^{*}(e)$ and $x$, where $e$ is the identity of $X$. We define $\alpha^{*}$ as $\alpha^{*}(x)=\pi \circ \alpha \circ \pi^{-1}(x)$. To show $\alpha^{*}$ is well defined consider $(x, y)$ and $\left(x, y^{\prime}\right)$ in $\pi^{-1}(x)$ and let $s_{\lambda}$ be a net with $(x, y) s_{\lambda} \rightarrow\left(x, y^{\prime}\right)$. Then $x s_{\lambda}=\pi\left((x, y) s_{\lambda}\right) \rightarrow \pi\left(x, y^{\prime}\right)=x$ and since $S$ is a subgroup of $X$ acting by right multiplication, $x^{\prime} s_{\lambda} \rightarrow x^{\prime}$ for every $x^{\prime}$ in $X$. Then

$$
\begin{aligned}
\pi(\alpha(x, y)) & =\lim \left[(\pi \circ \alpha(x, y)) s_{\lambda}\right] \\
& =\pi \circ \alpha\left(\lim (x, y) s_{\lambda}\right)=\pi \circ \alpha\left(x, y^{\prime}\right) .
\end{aligned}
$$

So $\alpha^{*}$ is well-defined and is clearly a homomorphism. Now for $x \in X$, $x=\lim s_{\mu}$ for some net in $S$, so $\alpha^{*}(x)=\alpha^{*}\left(\lim s_{\mu}\right)=\lim \alpha^{*}\left(s_{\mu}\right)=$ $\lim \left(\alpha^{*}(e)\right) s_{\mu}=\alpha^{*}(e) x$, where $e$ is the identity of $X$. That is the effect of $\alpha^{*}$ on $x$ is simply to multiply $x$ by $\alpha^{*}(e)$.

Note however this difficulty might be overcome by working with pointed transformation groups $\left(y_{0}, Y, T\right), y_{0} \in Y$, and associating it with $\left(\left(e, y_{0}\right), X \times Y, S\right)$; in this case $\alpha^{*}$ is the identity map on $X$ and the map $\alpha^{\prime}: Y \rightarrow W$ defined by $\alpha^{\prime}(y)=\pi_{2} \alpha(e, y)$ where $\pi_{2}$ is the projection onto the second coordinate, is a homomorphism of $(Y, T)$ onto $(W, T)$ since

$$
\begin{aligned}
\left(e, \alpha^{\prime}(y t)\right) & =\alpha(e, y t)=\alpha\left(\lim (e, y) x_{n}^{\prime}\right) \\
& =\lim \left((\alpha(e, y)) x_{n}^{\prime}\right)=\left(e,\left(\pi_{2}^{\circ} \alpha(e, y)\right) t\right) \\
& =\left(e, \alpha^{\prime}(y) t\right),
\end{aligned}
$$

where $x_{n}^{\prime}$ is a sequence in $S$ converging to $e$ and $t_{n}=t$ for all $x_{n}^{\prime}$.

We have showed the close relationship between the homomorphism $\varphi:(Y, T) \rightarrow(W, T)$ and the homomorphism $\psi:(X \times Y, S) \rightarrow(W \times$ $W, T)$ for some basic properties of homomorphisms. Below we mention some other properties that might be investigated. First we note that if $(Y, T)$ is a regular minimal set, then $\theta:(X \times Y, S) \rightarrow(X, S)$ is a regular homomorphism (see [8] and [9]). Also if $\varphi$ is a regular homomorphism, so is $\psi$. One might also define a $\tau$-topology on $(Y, T)$ and on the fibers of $\theta$ and define a group, $g\left(w_{0}\right)$, for factors $(W, T)$ of $(Y, T)$ as $\{y \in$ $\left.Y u: \varphi(y)=w_{0}\right\}$, where $u$ is an idempotent in the semigroup $Y$, such a group may correspond to a similarly defined group of $(X \times W, S)$ as a factor of $(X \times Y, S)$, [2]. One may also look at $p$-universal minimal set relative to a fixed minimal set and a property $p$ or at questions of relative disjointness [9].

\section{Section 3.}

EXAmPle 3.1. Proposition 2.4 permits us to give an example to a well known question. Let $W$ be a singleton and $Y$ be the circle acted on 
by two homeomorphisms, one an irrational rotation, $\alpha$, so $(Y, T)$ will be minimal, and the other, $\beta$, the map $y \rightarrow e^{2 \pi i^{2}}$, where $y=e^{2 \pi i r}$, so $(Y, T)$ will be proximal (i.e. $\varphi:(Y, T) \rightarrow(W, T)$ is proximal), $T$ is the subgroup of the group of homeomorphisms of $Y$ generated by the two homeomorphisms above. Then $\psi:(X \times Y, S) \rightarrow(X, S)=(X \times W, S)$ is proximal. So $(X \times Y, S)$ is proximally equicontinuous, $S$ is abelian, but $(X \times Y, S)$ is not locally almost periodic since it contains no distal points [5].

EXAmPLE 3.2. In this example $(Y, T)$ is equicontinuous but $(X \times$ $Y, S)$, as defined in $\S 2$, is not equicontinuous.

Let $Y=Z_{3}, T=Z$ and the action be defined by $(z, t) \rightarrow z+t \bmod 3$. Let the enumeration of

$$
T=\left\{t_{n}\right\}_{n=1}^{\infty} \text { be } t_{n}=\left\{\begin{array}{lll}
0 & \text { if } & n \equiv 0 \bmod 3 \\
1 & \text { if } & n \equiv 1 \bmod 3 \\
2 & \text { if } & n \equiv 2 \bmod 3
\end{array}\right.
$$

With $(X \times Y, S)$ as in $\S 2$, note

$$
(e, 0) x_{3 n+1}^{1} \rightarrow(e, 1)
$$

and

$$
(e, 1)\left(x_{3 n+1}^{1}\right)^{-1}=(e, 1) x_{3 n+1}^{1} \rightarrow(e, 2) ;
$$

we see $(e, 0)$ and $(e, 1) x_{3 n+1}^{1}$ are moved near $(e, 1)$ by $x_{3 n+1}^{1} \in S$, and so $(e, 0)$ and $(e, 2)$ are regionally proximal.

Note that the fibers of the projection $\pi:(X \times Y, S) \rightarrow(X, S)$ are finite, so this example shows that a finite-to-one extension of an equicontinuous minimal set may be distal without being equicontinuous even when the phase group is abelian. For the details involved in this question and for previous examples see [8] and [6]. In [11] Wu has shown that if a minimal set $(Y, T)$ is a finite-to-one extension of an equicontinuous minimal set with connected phase space, then $(Y, T)$ is equicontinuous (he proves this under some additional restrictions which are unnecessary).

In light of the role that connectedness plays in the above, the question occurs whether a proximally equicontinuous minimal set with abelian phase group and connected phase space would have to be locally almost periodic. To answer this question and to illustrate further the ideas of $\S 1$, we provide the following example.

EXAMPLE 3.3. Assuming the notation of $\S 1$ let $G_{n}=Y=$ unit circle in the complex plane and let $\alpha, \beta$ be as in Example 3.1. Define an action of $G_{n}$ on $G_{n} \times Y$ by $\left(g^{\prime}, y\right) g=\left(g^{\prime} g, y t_{n} g t_{n}^{-1}\right)$, where

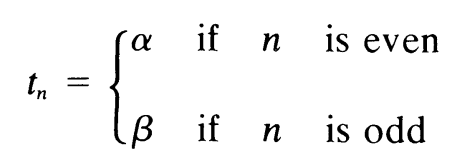


Form $(X \times Y, S)$ as in $\S 1$ and note that by taking $g \in G_{n}=Y$ such that $\alpha(y)=g y$ (multiplication) for every $y$, we see $\lim \left[(e, y) x_{2 n}^{g}\right]=(e, \alpha(y))$ and so $\left(e, \alpha^{n}(y)\right) \in \operatorname{cls}((e, y) S)$ for every $n$; thus $(X \times Y, S)$ is minimal since the projection $\pi$ of $(X \times Y, S)$ onto $(X, S)$ is open. Now to show that $\pi$ is a proximal homomorphism, we need only show that for $y, y^{\prime}$ in $Y,(e, y)$ and $\left(e, y^{\prime}\right)$ are proximal. Fix $y, y^{\prime} \in Y$ with $r^{\prime}>r$ where $y=e^{2 \pi i r}$, $y^{\prime}=e^{2 \pi i r^{\prime}}$; i.e. $y^{\prime}$ is on the arc going clockwise from $y$ to 1 .

Now $\beta^{-1}$ fixes 1 and moves all other points counter-clockwise while $\beta$ fixes 1 and moves all other points clockwise. Take $g$ such that $g \beta(y)=1$, then $\beta$ moves $y$ clockwise toward 1 with $y^{\prime}$ in between; $g$ rotates $\beta(y)$ and 1 to $g \beta(y)=1$ and $g$ with $g \beta\left(y^{\prime}\right)$ on the counter-clockwise arc from $g$ to 1 ; and $\beta^{-1}$ moves $g$ counter-clockwise toward $g \beta(y)$ with $g \beta\left(y^{\prime}\right)$ in between; in effect, $\beta^{-1}(g(\beta(y)))$ and $\beta^{-1}\left(g\left(\beta\left(y^{\prime}\right)\right)\right)$ are closer than $\beta(y)$ and $\quad \beta\left(y^{\prime}\right)$. Now $(e, y) x_{2 n+1}^{g} \rightarrow\left(e, \beta^{-1}(g(\beta(y)))\right) \quad$ and $\left(e, y^{\prime}\right) x_{2 n+1}^{g} \rightarrow\left(e, \beta^{-1}\left(g\left(\beta\left(y^{\prime}\right)\right)\right)\right)$. Since the $y$ and $y^{\prime}$ were fixed but arbitrary (i.e. $g$ depends on $y$ and $\left.y^{\prime}\right)$, it is clear that $(e, y)$ and $\left(e, y^{\prime}\right)$ are proximal.

Added in proof. $\quad$ B. Weiss and $\mathrm{H}$. Furstenberg now have an example of a minimal set with $X$ metric and $T$ the integers that is proximally equicontinuous but not locally almost periodic (personal communication).

\section{REFERENCES}

1. Joseph Auslander, B. Horelick, Regular minimal sets, II, the proximally equicontinuous case, Compositio Math., 22 (1970), 203-214.

2. R. Ellis, Lectures on topological dynamics, Math. Lecture Note Series, Benjamin, N.Y. 1969.

3. W. H. Gottschalk, An irreversible minimal set, Ergodic Theory, Proc. of an international symposium (New Orleans, 1961). Academic Press, New York, 1963, 121-134.

4. D. McMahon, Relativized problems with abelian phase group in topological dynamics, Proc. Nat Acad. of Sci., U.S.A. 73 No. 4, (1976).

5. - , T. S. Wu, On weak mixing and local almost periodicity, Duke Math. J., 39 (1972), 333-343.

6. _- On the connectedness of homomorphisms in topological dynamics, submitted.

7. K. Petersen, L. Shapiro, Induced flows, Trans. Amer. Math. Soc., 177 (1973), 375-390.

8. R. Sacker, G. Sell, Finite extensions of minimal transformation groups, Trans. Amer. Math. Soc., 190 (1974), 325-334.

9. P. Schoenfeld, Regular homomorphisms of minimal sets, Thesis, (University of Maryland, 1974).

10. T. S. Wu, Notes on topological dynamics, I. Relative disjointness, relative regularity and homomorphisms, Bull. Inst. Math. Acad. Sinica, 2 (1974), 343-356.

11. Notes on topological dynamics, II, Distal extensions with discrete fibers and prime flows, June 1975 Bull. Inst. Math. Acad. Sinica.

Received December 3, 1975.

UNIVERSITY OF OREGON

AND

New Mexico State University 


\section{PACIFIC JOURNAL OF MATHEMATICS}

\section{EDITORS}

RICHARD ARENS (Managing Editor)

University of California

Los Angeles, California 90024

\author{
R. A. Beaumont \\ University of Washington \\ Seattle, Washington 98105
}

\section{J. DugunduI}

Department of Mathematics University of Southern California Los Angeles, California 90007

D. Gilbarg and J. Milgram

Stanford University

Stanford, California 94305

\section{ASSOCIATE EDITORS}

E. F. BECKENBACH
B. H. NeumanN

F. Wolp
K. YoshidA

\section{SUPPORTING INSTITUTIONS}

UNIVERSITY OF BRITISH COLUMBIA

CALIFORNIA INSTITUTE OF TECHNOLOGY

UNIVERSITY OF CALIFORNIA

MONTANA STATE UNIVERSITY

UNIVERSITY OF NEVADA

NEW MEXICO STATE UNIVERSITY

OREGON STATE UNIVERSITY

UNIVERSITY OF OREGON

OSAKA UNIVERSITY

\author{
UNIVERSITY OF SOUTHERN CALIFORNIA \\ STANFORD UNIVERSITY \\ UNIVERSITY OF HAWAII \\ UNIVERSITY OF TOKYO \\ UNIVERSITY OF UTAH \\ WASHINGTON STATE UNIVERSITY \\ UNIVERSITY OF WASHINGTON \\ AMERICAN MATHEMATICAL SOCIETY
}

The Supporting Institutions listed above contribute to the cost of publication of this Journal, but they are not owners or publishers and have no responsibility for its contents or policies.

Mathematical papers intended for publication in the Pacific Journal of Mathematics should be in typed form or offset-reproduced (not dittoed). double spaced with large margins. Underline Greek letters in red, German in green, and script in blue. The first paragraph or two must be capable of being used separately as a synopsis of the entire paper. Items of the biblography should not be cited there unless absolutely necessary, in which case they must be identified by author and Journal, rather than by item number. Manuscripts, in duplicate, may be sent to any one of the four editors. Please classify according to the scheme of Math. Reviews, Index to Vol. 39. All other communications should be addressed to the managing editor, or Elaine Barth, University of California, Los Angeles, California, 90024.

100 reprints are provided free for each article, only if page charges have been substantially paid. Additional copies may be obtained at cost in multiples of 50 .

The Pacific Journal of Mathematics is issued monthly as of January 1966. Regular subscription rate: $\$ 72.00$ a year (6 Vols., 12 issues). Special rate: $\$ 36.00$ a year to individual members of supporting institutions.

Subscriptions, orders for back numbers, and changes of address should be sent to Pacific Journal of Mathematics, 103 Highland Boulevard, Berkeley, California, 94708.

PUBLISHED BY PACIFIC JOURNAL OF MATHEMATICS, A NON-PROFIT CORPORATION Printed at Jerusalem Academic Press, POB 2390, Jerusalem, Israel.

Copyright (C) 1976 Pacific Journal of Mathematics All Rights Reserved 


\section{Pacific Journal of Mathematics}

\section{Vol. 64, No. $2 \quad$ June, 1976}

Richard Fairbanks Arnold and A. P. Morse, Plus and times............. 297

Edwin Ogilvie Buchman and F. A. Valentine, External visibility ......... 333

R. A. Czerwinski, Bonded quadratic division algebras.............. 341

William Richard Emerson, Averaging strongly subadditive set functions in unimodular amenable groups. II .........................

Lynn Harry Erbe, Existence of oscillatory solutions and asymptotic behavior for a class of third order linear differential equations ............. 369

Kenneth R. Goodearl, Power-cancellation of groups and modules........ 387

J. C. Hankins and Roy Martin Rakestraw, The extremal structure of locally compact convex sets ...................................

Burrell Washington Helton, The solution of a Stieltjes-Volterra integral

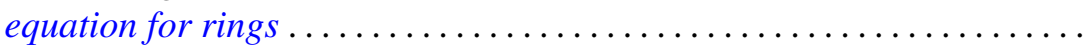

Frank Kwang-Ming Hwang and Shen Lin, Construction of 2-balanced

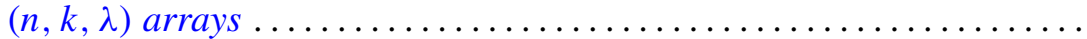

Wei-Eihn Kuan, Some results on normality of a graded ring ... 455

Dieter Landers and Lothar Rogge, Relations between convergence of series and convergence of sequences ......................... 465

Lawrence Louis Larmore and Robert David Rigdon, Enumerating immersions and embeddings of projective spaces ................

Douglas C. McMahon, On the role of an abelian phase group in relativized problems in topological dynamics..................

Robert Wilmer Miller, Finitely generated projective modules and TTF classes...

Yashaswini Deval Mittal, A class of isotropic covariance functions ...

Anthony G. Mucci, Another martingale convergence theorem ...

Joan Kathryn Plastiras, Quasitriangular operator algebras ...

John Robert Quine, Jr., The geometry of $p\left(S^{1}\right) \ldots \ldots \ldots$. 